\title{
INCIDENCE DE LA PANCREATIQUE CHRONIQUE DANS 8 DEPARTEMENTS FRANCAIS
}

\author{
J. FAIVRE ${ }^{(1)}$ - D. PILLON ${ }^{(1)}$
}

Après 3 ans d'enregistrement systématique des cas de pancréatite chronique (1990 - 1992) dans 8 départements, il est possible de faire une première analyse de l'incidence de cette pathologie.

Les données du recensement de 1990 fournies par l'INSEE ont permis de calculer les taux d'incidence. Les taux annuels et les taux spécifiques pour l'âge ont été déterminés sur la moyenne des trois années d'enregistrement. Pour comparer l'incidence entre des départements de structures d'âge différents, les taux ont été standardisés pour l'âge en utilisant la population mondiale de référence. Une estimation de l'incidence pour la France entière et du nombre des nouveaux cas annuels a été réalisée en faisant l'hypothèse que l'incidence moyenne de ces 8 départements était représentative de celle de la France entière.

Chez l'homme les taux d'incidence pour 100.000 habitants standardisés selon la population européenne était de 9,1 dans le Calvados, 10,6 en Côte d'Or, 9,4 dans les Hautes-Pyrénées, 6,1 dans l'Oise, 5,9 dans les Pyrénées Atlantiques, 8,6 dans la Somme, 7,6 dans le Var et 7,5 en Vendée. Chez les femmes les taux étaient respectivement de 1,$1 ; 2,3 ; 3,0 ; 1,0 ; 0,8 ; 1,1$; 1,$8 ; 0,8$ et 1,4 . Le sex ratio variait entre 4,6 et 9,5 . L'incidence de la pancréatite chronique chez l'homme augmente avec l'âge jusqu'à 35 ans puis se stabilise. Après 35 ans les taux d'incidence sont voisins dans tous les groupes d'âge. Chez la femme les taux d'incidence sont voisins dans toutes les tranches d'âge.

Le taux estimé d'incidence brut pour la France entière était de 7,7/100.000 chez l'homme et de $1,2 / 100.000$ chez la femme. Les taux d'incidence correspondant standardisés selon la population européenne étaient de 7,9 et 1,2. Le nombre estimé de nouveaux cas annuels étaient de 2120 chez l'homme et de $350 \mathrm{chez}$ la femme.

Ces résultats doivent être considérés comme des résultats préliminaires. Ils seront légèrement modifiés lorsque tous les contrôles d'exhaustivité en cours seront disponibles.

(1) Hôpital Général (Dijon)

\section{PREVALENCE DE LA PANCREATITE CHRONIQUE}

\author{
A. VALLA ${ }^{(1)}-$ T. DAO $(1)$
}

La prévalence prend en compte tous les cas recensés pendant la période d'étude, indépendamment du début de la maladie. Elle cumule donc les anciens cas. Les nouveaux cas sont ceux dont le diagnostic a été évoqué pour la première fois pendant la durée de l'étude (entre le ler janvier 1990 et le 31 décembre 1992) : ils ont fourni les données d'incidence. Les anciens cas sont ceux pour lesquels le diagnostic de pancréatite a été évoqué pour la première fois avant le ler janvier 1990.

(1) Centre Hospitalier Universitaire (Caen) 